\title{
The impact of high-dose methotrexate on intracellular 6-mercaptopurine disposition during interval therapy of childhood acute lymphoblastic leukemia
}

\author{
T. Adam de Beaumais - T. Dervieux • M. Fakhoury \\ Y. Medard · S. Azougagh · D. Zhang · K. Yakouben • \\ E. Jacqz-Aigrain
}

Received: 27 August 2009 / Accepted: 27 November 2009 / Published online: 23 December 2009

(C) Springer-Verlag 2009

\begin{abstract}
Purpose Low-dose methotrexate (MTX) therapy is the cornerstone treatment of acute lymphoblastic leukemia (ALL) and may enhance the activation of 6-mercaptopurine (6-MP) to 6-thioguanine nucleotides (6-TGN). Yet, data have established that high-dose MTX (HDMTX) hampers the accumulation of 6-TGN in red blood cells (RBC) and lymphoblasts.

Methods To clarify the pharmacokinetic interactions between these two antimetabolites, we serially measured RBC 6-TGN and MTX polyglutamates (MTXPG) levels following repeated courses of HDMTX ( $5 \mathrm{~g} / \mathrm{m}^{2}$ over $24 \mathrm{~h}$ ) with daily oral 6-MP $\left(25 \mathrm{mg} / \mathrm{m}^{2}\right)$ during interval therapy in 20 children with ALL.

Results HDMTX produced a rapid reduction in RBC 6TGN $24 \mathrm{~h}$ after the start of MTX, and this effect was sustained at least by the third day (median decrease $-21 \%$; $P<0.001)$. However, a return to pre-infusion of 6-TGN levels was observed by the time of the following HDMTX course 14 days later $(P<0.001)$. RBC MTX polyglutamates accumulation followed Michaelis-Menten kinetics but was not associated with the change in pre-infusion
\end{abstract}

T. Adam de Beaumais · M. Fakhoury · Y. Medard · S. Azougagh . D. Zhang $\cdot$ E. Jacqz-Aigrain ( $\square)$

Department of Pediatric Pharmacology and Pharmacogenetics,

Robert Debre Hospital, 48 Boulevard Serurier,

75019 Paris, France

e-mail: evelyne.jacqz-aigrain@ rdb.aphp.fr

T. Dervieux

Cypress Bioscience, San Diego, CA, USA

K. Yakouben

Department of Hematology,

Robert Debre Hospital, Paris, France
6-TGN levels which remained stable during the interval period.

Conclusion HDMTX does not appear to enhance 6-MP activation to 6-TGN. Moreover, given that the deleterious effect of HDMTX on intracellular 6-MP disposition has been shown to be several folds greater in lymphoblasts than in RBC. Our data warrant additional studies elucidating the optimal MTX dose synergizing with 6-MP.

Keywords Methotrexate $\cdot$ Methotrexate polyglutamates · Mercaptopurine $\cdot$ Leukemia $\cdot$ Thioguanine nucleotides

\section{Introduction}

The optimization of antileukemic treatment regimens has considerably improved the outcome of childhood acute lymphoblastic leukemia (ALL), and the most recent TOTAL XV protocol has reported a 5-year survival rate greater than $90 \%$ [1]. Nonetheless, while these data are very encouraging, the incidence of relapse in ALL is still significant, and additional efforts to optimize the sequence, dosing, and schedule of administration of antileukemic agents will be essential to improve the survival of patients with high risk ALL and poor prognosis.

One of the cornerstone treatments of ALL is the combination of 6-mercaptopurine (6-MP) with low-dose methotrexate (MTX), and the observation that both these anticancer agents produce greater antileukemic effects than either treatment alone can be traced back nearly half a century ago [2]. 6-MP is a prodrug activated to 6-thioguanine nucleotides (6-TGN) by a multistep process involving several enzymes of the purine salvage pathway including hypoxanthine guanine phosphoribosyl transferase (HPRT), an anabolic route in competition with a thiopurine 
$S$-methyltransferase (TPMT)- and xanthine oxidase (XO)mediated inactivation of 6-MP to 6-methylmercaptopurine (6-MMPN) and 6-thiouric acid, respectively [3]. Alternatively, MTX is an antifolate producing antileukemic effects through an accumulation to long-chain MTXPG that potently inhibit de novo purine, tetrahydrofolate, and thymidylate biosynthesis [4].

The biochemical and pharmacological basis to combine 6-MP and MTX stem from various in vivo and in vitro observations whereby de novo purine biosynthesis inhibition by MTXPG produces an elevation in cellular phosphoribosylpyrophosphate (PPPR) levels [5, 6], the cofactor for HPRT. Hence, the combination of MTX with 6-MP is synergistic in enhancing the activation of 6-MP to 6-TGN that are incorporated to nucleic acids. Furthermore, by inhibiting of XO [7], MTX produces an increase in 6-MP peak plasma concentrations and area under the curve [8]. Arguably, the rational to combine MTX with 6-MP is sound, and in vivo data have established that greater accumulation of red blood cells (RBC) MTXPG are associated with greater RBC 6-TGN levels during maintenance therapy of ALL with weekly low-dose MTX (i.e., $40 \mathrm{mg} / \mathrm{m}^{2}$ ) and daily oral 6 -MP $\left(75 \mathrm{mg} / \mathrm{m}^{2}\right)$ [9]. Yet, the premise that MTX synergizes with 6-MP is somewhat challenged by recent data indicating that high MTX dosage (e.g., $1 \mathrm{~g} / \mathrm{m}^{2}$ ) hampers 6TGN accumulation following IV 6-MP administration ( $1 \mathrm{~g} /$ $\mathrm{m}^{2}$ ) during upfront treatment of ALL [10].

In the present study, we sought to clarify the relative contribution of HDMTX on 6-MP intracellular pharmacokinetics during interval therapy in children who received consecutive intravenous HDMTX courses $\left(5 \mathrm{~g} / \mathrm{m}^{2}\right.$ over 24 h every 14 days) with daily $6-\mathrm{MP}\left(25 \mathrm{mg} / \mathrm{m}^{2}\right)$.

\section{Methods}

Twenty children with ALL were enrolled in the European Organization for Research and Treatment of Cancer (EORTC) 58951 protocol. The protocol was divided into five treatment phases including induction (with a prephase), consolidation, interval, late intensification, and maintenance. Interval therapy started 77 days after induction and consisted of daily oral 6-MP $\left(25 \mathrm{mg} / \mathrm{m}^{2}\right)$ for 56 days together with three or four intravenous HDMTX $\left(5 \mathrm{~g} / \mathrm{m}^{2}\right)$ courses at 14-days interval (days 8, 22, 36, and 50) depending on the risk group allocation at diagnostic (very high risk patients received only three HDMTX courses). Local institutional review committee approved the study, and informed consent from parents or guardians was collected for all children. HDMTX courses consisted of $500 \mathrm{mg} / \mathrm{m}^{2}$ MTX infused over the first hour followed by a continuous infusion of $4,500 \mathrm{mg} / \mathrm{m}^{2}$ MTX over the remaining $23 \mathrm{~h}$. Folinic acid rescue therapy $\left(12 \mathrm{~g} / \mathrm{m}^{2}\right)$ was administered every $6 \mathrm{~h}$, starting $36 \mathrm{~h}$ after the beginning of infusion and given until MTX plasma levels were lower than $0.2 \mu \mathrm{M}$. Biochemical and hematological monitoring was carried out at each course. Grading scale used for adverse events evaluation was assessed using NCI CTC v3 criteria. Compliance to daily 6-MP treatment was assessed by study nurses.

During each course, MTX plasma concentrations were monitored at 24, 48, and $72 \mathrm{~h}$ after the start of MTX infusion using standard enzyme multiplied immunoassay technique (EMIT) (Cobas Mira $^{\mathrm{TM}}$, Roche laboratories). Similarly, RBC MTX polyglutamates (MTXPG, up to the pentaglutamate) and RBC 6-MP metabolites concentrations (6-TGN and 6-MMPN nucleotides) were measured before MTX infusion $(0 \mathrm{~h})$ and at 24, 48, and $72 \mathrm{~h}$ using validated liquid chromatography methods as described $[11,12]$ and reported as pmol/10 $10^{9} \mathrm{RBC}$ for MTXPG and $\mathrm{pmol} / 8 \times 10^{8}$ for 6 -MP metabolites. RBC MTXPG levels were categorized as short-chains MTXPG $\left(\mathrm{MTXPG}_{1-2}\right.$ as the sum of MTXPG $_{1}$ with MTXPG $_{2}$ ), long-chain MTXPG $\left(\mathrm{MTXPG}_{3}\right)$, or very long-chains MTXPG $\left(\mathrm{MTXPG}_{4-5}\right.$ as the sum of $\mathrm{MTXPG}_{4}$ with $\mathrm{MTXPG}_{5}$ ). Total MTXPG levels were calculated as the sum of all MTXPG species $\left(\mathrm{MTXPG}_{1-5}\right)$. Statistical analysis was carried out using Statistica (Statsoft, Tulsa, OK). The kinetics of MTXPG accumulation was assessed using GraphPad Prism 4 software (GraphPad, La Jolla, CA). Pairwise differences between 6-TGN and 6-MMPN levels were analyzed using Wilcoxon's test, and correlations were determined using Spearman rank test.

\section{Results}

A total of 20 children ( 13 boys and 7 girls) with ALL aged 2-15 (mean: $6.35 \pm 3.42 \mathrm{y}$, average $\pm \mathrm{SD}$ ) were enrolled. Fifteen patients presented with B-ALL, and five patients presented T-ALL. A total of 78 HDMTX courses were administered, 18 patients received four courses HDMTX, and two patients received three courses (one patient relapsed after the third course, and one patient with very high risk ALL received three courses per protocol). Blood for RBC 6-thiopurine nucleotides (6-TGN and 6-MMPN) or RBC MTXPG measurements was available in 76 of these 78 courses. Creatinine clearance was $127 \pm 22 \mathrm{ml} /$ $\min (n=20)$ at the start of the first course, and renal function remained normal during interval therapy (not shown). No significant hematological or biochemical toxicities occurred during the study period, and none of the patients required discontinuation of or alteration in 6-MP therapy. Three grade 3-4 adverse effects occurred in three patients (two infections and one meningitis relapse).

Plasma and RBC MTX concentrations during the first HDMTX course (day 8) are presented in Fig. 1a, b. RBC 

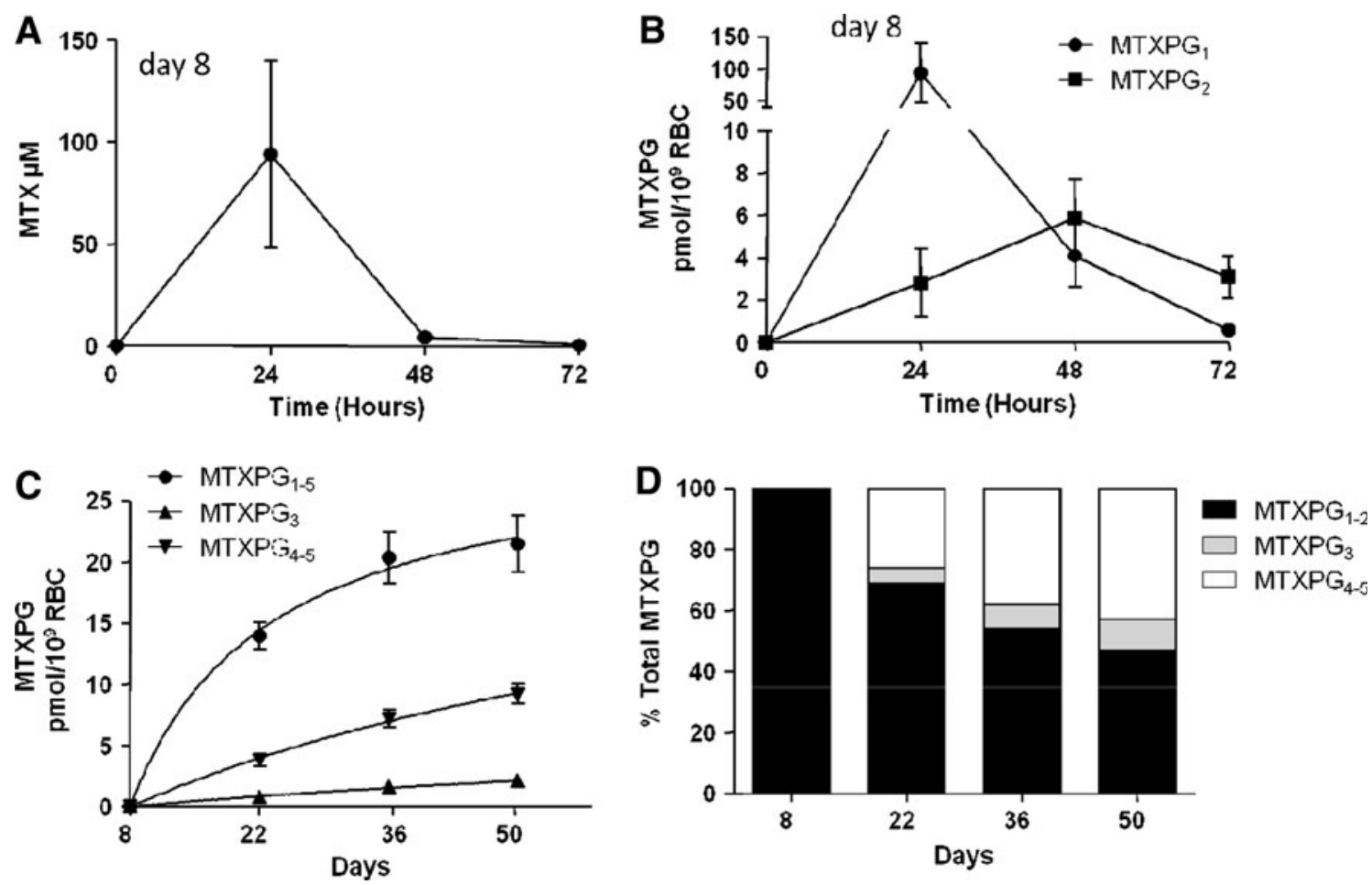

Fig. 1 Methotrexate pharmacokinetics and MTXPG accumulation in children with ALL receiving HDMTX. Error bars represent standard deviations (SD). a Changes in MTX plasma concentrations during the first HDMTX infusion (day 8), b change in RBC MTXPG 1 (MTX) and $\mathrm{MTXPG}_{2}$ during the first HDMTX infusion (day 8). $\mathrm{MTXPG}_{3-5}$ levels were undetectable, $\mathrm{c}$ accumulation of RBC MTXPG during interval

therapy. HDMTX was administered at days 8, 22, 36, and 50 in 17 patients. Median MTXPG $_{1-5}$ levels at day 50 were $20 \mathrm{pmol} / 10^{9}$ cells (IQ range $15-29 \mathrm{pmol} / 10^{9} \mathrm{RBC}$ ). Median $\mathrm{MTXPG}_{5}$ levels were $9 \mathrm{pmol} /$ $10^{9}$ cells (IQ range $7-11 \mathrm{pmol} / 10^{9} \mathrm{RBC}$ ), $\mathrm{d}$ change in the distribution of short chains $\left(\mathrm{MTXPG}_{1-2}\right)$ long chains $\left(\mathrm{MTXPG}_{3}\right)$ and very long chains $\left(\mathrm{MTXPG}_{4-5}\right)$ MTXPG during interval therapy

$\mathrm{MTXPG}_{1}$ (MTX) concentrations rapidly increased and then decreased in parallel to plasma MTX concentrations. Low albeit significant MTX di-glutamate $\left(\mathrm{MTXPG}_{2}\right)$ formation was detected in circulating erythrocytes during the first HDMTX course, but MTXPG with greater than two glutamic residues were undetectable. Within each subsequent course, there was no significant change in $\mathrm{RBC} M \mathrm{MTXPG}_{3-5}$ levels from pre-infusion levels to $72 \mathrm{~h}$ (not shown). Thus, total $\mathrm{MTXPG}_{1-5}$ concentrations were calculated as the sum of pre-infusion values for $\mathrm{MTXPG}_{1-2}$ plus the average $\mathrm{RBC}$ $\mathrm{MTXPG}_{3}$ and RBC MTXPG ${ }_{4-5}$ measured from 0 to $72 \mathrm{~h}$ within each course. Figure 1c, d highlights the accumulation of RBC MTXPG in 17 patients (in one patient, RBC MTXPG levels were available at day 50). The changes in MTXPG $_{1-5}$ over the interval period were best described by Michaelis-Menten kinetics (coefficient of determination ranged from 0.74 to 0.99 ) and with apparent plateau in total MTXPG by the 4th HDMTX infusion (6-weeks after the 1 st infusion). In contrast, RBC MTXPG 3 and $\mathrm{MTXPG}_{4-5}$ concentrations increased linearly $\left(\mathrm{R}^{2}\right.$ ranged from 0.71 to 0.99) with a selective redistribution of MTXPG toward long-chain $\mathrm{MTXPG}_{4-5}$ which represented over $40 \%$ of total MTXPG at day 50 (Fig. 1d).

The impact of HDMTX on RBC thiopurine nucleotides concentrations following 76 HDMTX in 20 patients is presented in Table 1 and revealed a rapid decrease in RBC 6TGN and 6-MMPN levels (nadir) within $24 \mathrm{~h}$ hours of HDMTX (compared to pre-HDMTX levels at $0 \mathrm{~h}$; $P<0.001)$ and lasting for a minimum of 3 days. However, this reduction was followed by a return to baseline levels prior to the initiation of the following HDMTX course 14 days later (6-TGN: $114 \pm 55$ versus $184 \pm 82 \mathrm{pmol} /$ $8 \times 10^{8}$ RBC; 6-MMPN: $1003 \pm 985$ versus $1351 \pm$ $1307 \mathrm{pmol} / 8 \times 10^{8} \mathrm{RBC} ; \quad P<0.001, n=60$ ) (average \pm $\mathrm{SD})$. The change in RBC 6-TGN or 6-MMPN levels from initiation of HDMTX infusion to 72-h post-infusion was not associated with concomitant MTX peak plasma levels or RBC MTXPG concentrations ( $P>0.09$; not shown). A lack of greater activation of 6-MP to intracellular metabolites by consecutive HDMTX was evident (Fig. 2). A high inter-individual variability of RBC 6-thiopurine nucleotide levels was observed. No significant difference between preinfusion levels measured after 8 days 6-MP (6-TGN: $177 \pm 81 \mathrm{pmol} / 8 \times 10^{8} \mathrm{RBC}$; 6-MMPN: $1522 \pm 1326 \mathrm{pmol} /$ $8 \times 10^{8} \mathrm{RBC}$ ) versus those measured after 22 days (6-TGN: $167 \pm 82 \mathrm{pmol} / 8 \times 10^{8} \mathrm{RBC}$; 6-MMPN: $1338 \pm 1101 \mathrm{pmol} /$ 
Table 1 Median (interquartile range) pre-HDMTX infusion RBC 6-TGN and 6-MMPN levels and percentage change within the first $72 \mathrm{~h}$ of initiation of HDMTX

\begin{tabular}{llllll}
\hline & $\begin{array}{l}0 \mathrm{~h} \\
\mathrm{pmol} / 8 \times 10^{8} \mathrm{RBC} \\
(N=76)\end{array}$ & $\begin{array}{l}24 \mathrm{~h} \\
(N=75)\end{array}$ & $\begin{array}{l}48 \mathrm{~h} \\
(N=70)\end{array}$ & $\begin{array}{l}72 \mathrm{~h} \\
(N=66)\end{array}$ & $\begin{array}{l}\text { nadir level 24-72 h } \\
(N=76)\end{array}$ \\
\hline $6-T G N$ & 176 & $-11 \%$ & $-22 \%$ & $-21 \%$ & $-35 \%$ \\
& $(128$ to 227$)$ & $(-31 \text { to } 15 \%)^{\dagger}$ & $(-38 \text { to }-6)^{\dagger}$ & -37 to $-3 \%)^{\dagger}$ & $(-49 \text { to }-19 \%)^{\dagger}$ \\
$6-M M P N$ & 939 & $-12 \%$ & $-19 \%$ & $-24 \%$ & $-31 \%$ \\
& $(625$ to 1613$)$ & $(-33 \text { to } 18 \%)^{\dagger}$ & $(-3 \% \text { to } 2 \%)^{\dagger}$ & $(-40 \text { to }-11 \%)^{\dagger}$ & $(-45 \text { to }-15 \%)^{\dagger}$ \\
\hline
\end{tabular}

Pre-HDMTX infusion of 6-thiopurine nucleotide levels was available in 76 of 78 HDMTX courses administered

$\dagger P<0.001$ versus pre-infusion levels

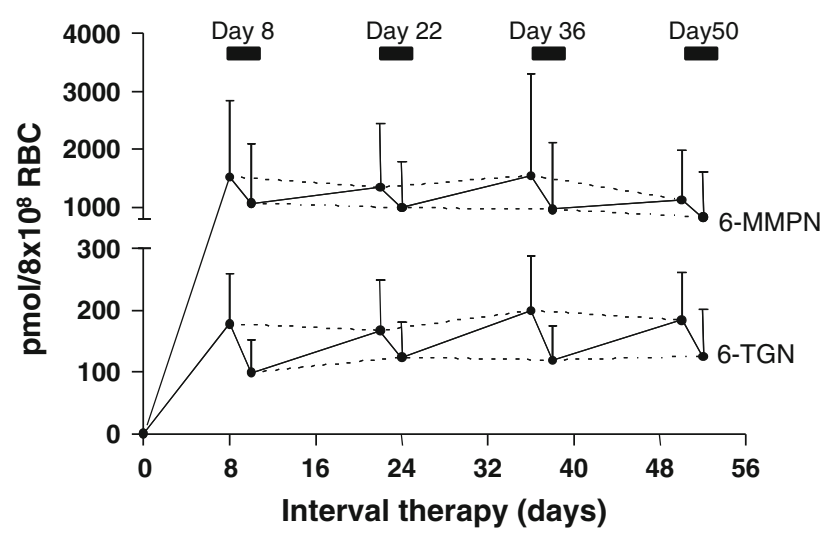

Fig. 2 Impact of HDMTX infusions on 6-thiopurine disposition in RBC. Average pre-HDMTX infusion of RBC 6-TGN and 6-MMPN levels and nadir concentrations within 72-h post-infusion measured from day 8 to day 50 of interval therapy. Error bars represent standard deviations (SD). Dotted line corresponds to baseline and trough 6TGN and 6-MMPN concentrations. Days of HDMTX infusion are indicated

$8 \times 10^{8}$ RBC), 36 days $\left(6-\mathrm{TGN}: 199 \pm 87 \mathrm{pmol} / 8 \times 10^{8}\right.$ RBC; 6-MMPN: $\left.1544 \pm 1755 \mathrm{pmol} / 8 \times 10^{8} \mathrm{RBC}\right)$, and 50 days (6-TGN: $184 \pm 76 \mathrm{pmol} / 8 \times 10^{8} \mathrm{RBC}$; 6-MMPN: $1126 \pm 848 \mathrm{pmol} / 8 \times 10^{8} \mathrm{RBC}$ ) (average $\pm \mathrm{SD} ; P>0.14$ ).

\section{Discussion}

This study is the first to describe the build-up of MTXPG in the erythrocytic compartment following consecutive HDMTX courses and the second report to suggest a negative impact of HDMTX on 6-MP intracellular metabolism [10].

MTX is activated to MTXPG by a FPGS-mediated sequential addition of glutamic residues on MTX, and several studies have established that MTXPG accumulation in leukemic blasts or surrogate erythrocytes is associated with antileukemic effects $[13,14]$. Our data indicate that the accumulation of $\mathrm{MTXPG}_{1-5}$ following consecutive HDMTX was slow, and the apparent plateau approaching steady state by the 6th-week therapy is consistent with previous reports with low weekly MTX dosage $[15,16]$. During the first HDMTX course, the kinetics of RBC MTXPG (parent methotrexate) mirrored those of plasma MTX, and no MTXPG with greater than two glutamic residue was detected. These data substantiate the notion that the circulating erythrocytes do not produce significant MTXPG, and the low, albeit significant MTX di-glutamate formation observed during the first infusion probably reflected low polyglutamation from newly released reticulocytes from bone marrow. Alternatively, the subsequent elevation in RBC $\mathrm{MTXPG}_{3-5}$ likely reflected the pulsative release of MTXPG incorporated in bone marrow erythroid progenitors. Interestingly, the median RBC MTXPG ${ }_{1-5}$ levels measured at the initiation of the last HDMTX course was $20 \mathrm{pmol} / 10^{9}$ cells, and these values are similar to those reported in RBC from children receiving weekly MTX dosages 125 -fold lower $\left(40 \mathrm{mg} / \mathrm{m}^{2}: 29 \mathrm{pmol} / 10^{9}\right.$ cells) [9]. We explain these results by rate-limiting FPGS activity in marrow progenitors rather than saturation of MTX uptake (which is passive at higher dose MTX). However, HDMTX administration resulted in fourfold higher $\mathrm{MTXPG}_{5}$ levels (median $9.2 \mathrm{pmol} / 10^{9}$ cells) by comparison with very longchain MTXPG levels observed during weekly low-dose MTX during maintenance (median MTXPG $_{5-7}$ was $2.4 \mathrm{pmol} / 10^{9}$ cells) [9], and these data are consistent with the greater accumulation of long-chain MTXPG in bone marrow lymphoblasts following higher dose MTX [17]. Moreover, the percentage of very long-chain $\mathrm{MTXPG}_{4-5}$ (over $40 \%$ of the total MTXPG) following HDMTX courses was significantly higher than those reported following maintenance therapy of ALL (i.e., $18 \% \mathrm{MTXPG}_{4-5}$ with a $20 \mathrm{~g} / \mathrm{m}^{2}$ dosage) [9, 18]. Altogether, these data support the rational of administering HDMTX to produce longer chain MTXPG which are more potent inhibitors of de novo purine biosynthesis than short-chains MTXPG $[17,19]$.

The studies that directly investigated the impact of MTX on 6-thiopurine nucleotides (6-TGN and 6-MMPN) accumulation are in vivo very limited $[9,10]$, and the only 
trial that was specifically design to address the synergism between HDMTX and 6-MP metabolism (TOTAL XIIIb) surprisingly revealed that HDMTX (e.g., $1 \mathrm{~g} / \mathrm{m}^{2}$ ) hampered the accumulation of intracellular 6-thiopurine nucleotides in bone marrow blasts, circulating blasts, and erythrocytes following IV 6-MP during upfront treatment of ALL [10]. In our study, the impact of HDMTX on 6-MP intracellular disposition was assessed in erythrocytes after a minimum of 8 days of continuous daily 6-MP, and thus at steady state concentration [20]. Our data consolidate and provide a valuable complement of the previous unexpected finding that HDMTX can negatively impact the intracellular metabolism of 6-MP. We observed a $\sim 25 \%$ reduction in RBC 6-TGN and 6-MMPN levels within the first $72 \mathrm{~h}$ following the initiation of HDMTX with oral daily 6-MP, an effect remarkably similar to those reported in TOTALXIIIb (6-TGN: $-35 \%$; 6-MMPN: $-29 \%) 20 \mathrm{~h}$ after initiation of intravenous 6-MP. However, we establish that the rapid reduction in RBC 6-MP metabolites by HDMTX which was sustained at least by the 3rd day post-HDMTX was followed by a recovery by the time of the next HDMTX infusion (14 days later). Several explanations were set forth [10] to explain the antagonism of HDMTX on 6-MP intracellular metabolism in TOTAL XIIIb, despite the significant de novo purine biosynthesis inhibition by MTX [17] and hence the likely elevation in PRPP levels [6]. In particular, an alteration in 6-MP uptake following tumor cell lysis and release of hypoxanthine (that competes with 6-MP for HPRT) was proposed, but this primary mechanism of cell lysis cannot hold given that our children were in remission [10]. We believe that alternative mechanisms including a selective efflux of 6-TGN and 6-MMPN is more likely to explain the results of this study and our.

It is important to note that in vitro studies in leukemic cell lines have reported an opposite effects of MTX on 6TGN accumulation ranging from synergism at low-dose MTX ( twofold higher 6-TGN following $0.02 \mu \mathrm{M}$ MTX) [21] to antagonism at much higher dosage ( $\sim 2$ lower fold higher 6-TGN following $40 \mu \mathrm{M}$ MTX) [22], and it is tempting to suggest that MTX dose may produce differential effects on 6-TGN accumulation in vivo. Our contention is supported by the observation that low-dose MTX can enhance 6-TGN accumulation as seen during continuation therapy of ALL [9]. In addition, should HDMTX only transiently decrease 6-TGN levels by the time of its administration but in fine synergize with repetitive daily 6MP dose, we should have observed an elevation in preinfusion 6-TGN levels during the interval period. As we have not, we speculate that HDMTX does not synergize with 6-MP, while lower MTX dosage may enhance 6-MP activation to 6-thiopurine nucleotides. Whether these observations are clinically relevant is not know, but the reduction in 6-TGN accumulation in bone marrow lym- phoblasts following HDMTX with intravenous 6-MP has been reported to be significantly greater $(\sim 13$ fold $)$ than those observed in red cells ( $\sim 1.5$ fold) during upfront therapy of ALL [10], and lower RBC 6-TGN are associated with lower antileukemic effects during maintenance of ALL $[13,23]$. Clearly, these data cannot be ignored, and mechanisms of interaction between 6-MP and MTX are important to identify, not only to increase our knowledge on the transport, metabolism, and action of these drugs, but also to open new ways for treatment optimization and individualization.

Our data warrant additional studies assessing the duration of thioguanine levels suppression, the impact on their incorporation into DNA, the tolerance of increasing 6-MP dosage during HDMTX courses. Moreover, 6-MP and MTX transporters polymorphisms and/or expression might participate to inter-individual variability in both 6-thioguanine and MTXPG concentrations and also impact 6-TGN decrease. These investigations could help determine the dosing regimen for optimal clinical efficacy during interval therapy.

\section{References}

1. Pui CH, Campana D, Pei D, Bowman WP, Sandlund JT, Kaste SC et al (2009) Treating childhood acute lymphoblastic leukemia without cranial irradiation. N Engl J Med 360:2730-2741

2. Frei E III, Freireich EJ, Gehan E, Pinkel D, Holland JF et al (1961) Studies of sequential and combination antimetabolite therapy in acute lymphoblastic leukemia: 6-mercaptopurine and methotrexate. Blood 18:431-453

3. Lennard L (1992) The clinical pharmacology of 6-mercaptopurine. Eur J Clin Pharmacol 43:329-339

4. Gorlick R, Goker E, Trippett T, Waltham M, Banerjee D, Bertino JR (1996) Intrinsic and acquired resistance to methotrexate in acute leukemia. N Engl J Med 335:1041-1048

5. Bokkerink JP, Bakker MA, Hulscher TW, De Abreu RR, Schretlen ED, van Laarhoven JP, De Bruyn CH (1986) Sequence-, time- and dose-dependent synergism of methotrexate and 6-mercaptopurine in malignant human T-lymphoblasts. Biochem Pharmacol 35:3549-3555

6. Tan CT, Wollner N, Trippett T, Goker E, Tong WP, Kheradpour A et al (1994) Pharmacologic-guided trial of sequential methotrexate and thioguanine in children with advanced malignancies. J Clin Oncol 12:1955-1962

7. Lewis AS, Murphy L, McCalla C, Fleary M, Purcell S (1984) Inhibition of mammalian xanthine oxidase by folate compounds and amethopterin. J Biol Chem 259:12-15

8. Balis FM, Holcenberg JS, Zimm S, Tubergen D, Collins JM, Murphy RF, Gilchrist GS, Hammond D, Poplack DG (1987) The effect of methotrexate on the bioavailability of oral 6-mercaptopurine. Clin Pharmacol Ther 41:384-387

9. Dervieux T, Hancock M, Evans W, Pui CH, Relling MV (2002) Effect of methotrexate polyglutamates on thioguanine nucleotide concentrations during continuation therapy of acute lymphoblastic leukemia with mercaptopurine. Leukemia 16:209-212

10. Dervieux T, Hancock ML, Pui C-H, Rivera GK, Sandlund JT, Ribeiro R, Boyett J, Evans WE, Relling MV (2003) Antagonism of methotrexate on mercaptopurine disposition in lymphoblasts 
during up-front treatment of acute lymphoblastic leukemia. Clin Pharmacol Ther 73:506-516

11. Dervieux T, Boulieu R (1998) Simultaneous determination of 6-thioguanine and methyl 6-mercaptopurine nucleotides of azathioprine in red blood cells by HPLC. Clin Chem 44:551-555

12. Dervieux T, Orentas LD, Marcelletti J, Pischel K, Smith K, Walsh M, Richerson R (2003) HPLC determination of erythrocyte methotrexate polyglutamates after low-dose methotrexate therapy in patients with rheumatoid arthritis. Clin Chem 49:1632-1641

13. Schmiegelow K, Schroder H, Gustafsson G, Kristinsson J, Glomstein A, Salmi T, Wranne L (1995) Risk of relapse in childhood acute lymphoblastic leukemia is related to RBC methotrexate and mercaptopurine metabolites during maintenance chemotherapy. Nordic society for pediatric hematology and oncology. J Clin Oncol 13:345-351

14. Masson E, Relling MV, Synold TW, Liu Q, Schuetz JD, Sandlund JT, Pui CH, Evans WE (1996) Accumulation of methotrexate polyglutamates in lymphoblasts is a determinant of antileukemic effects in vivo. A rationale for high- dose methotrexate. J Clin Invest 97:73-80

15. Schroder H, Clausen N, Ostergaard E, Pressler T (1986) Pharmacokinetics of erythrocyte methotrexate in children with acute lymphoblastic leukemia during maintenance treatment. Cancer Chemother Pharmacol 16:190-193

16. Hendel J, Nyfors A (1984) Pharmacokinetics of methotrexate in erythrocytes in psoriasis. Eur J Clin Pharmacol 27:607-610
17. Dervieux T, Brenner TL, Hon YY, Zhou Y, Hancock ML, Sandlund JT et al (2002) De novo purine synthesis inhibition and antileukemic effects of mercaptopurine alone or in combination with methotrexate in vivo. Blood 100:1240-1247

18. Schroder H, Fogh K, Schrder H (1988) Methotrexate and its polyglutamate derivatives in erythrocytes during and after weekly low-dose oral methotrexate therapy of children with acute lymphoblastic leukemia. Cancer Chemother Pharmacol 21:145-149

19. Allegra CJ, Drake JC, Jolivet J, Chabner BA (1985) Inhibition of phosphoribosylaminoimidazolecarboxamide transformylase by methotrexate and dihydrofolic acid polyglutamates. Proc Natl Acad Sci U S A 82:4881-4885

20. Rostami-Hodjegan A, Lennard L, Lilleyman JS (1995) The accumulation of mercaptopurine metabolites in age fractionated red blood cells. Br J Clin Pharmacol 40:217-222

21. Giverhaug T, Loennechen T, Aarbakke J (1998) Increased concentrations of methylated 6-mercaptopurine metabolites and 6-thioguanine nucleotides in human leukemic cells in vitro by methotrexate. Biochem Pharmacol 55:1641-1646

22. Innocenti F, Danesi R, Di Paolo A, Loru B, Favre C, Nardi M, Macchia P, Del Tacca M (1996) Clinical and experimental pharmacokinetic interaction between 6-mercaptopurine and methotrexate. Cancer Chemother Pharmacol 37:409-414

23. Lennard L, Lilleman JS (1989) Variable mercaptopurine metabolism and treatment outcome in childhood lymphoblastic leukemia. J Clin Oncol 7:1816-1823 